\title{
Ausschreibungen der AO
}

\author{
Georg Duda
}

\section{AO Research Institute (ARI) Medical Research Fellowship 2018}

Die ARI Fellowship ermöglicht Ärzten am AO-Forschungsinstitut Erfahrungen in der angewandten Forschung zu sammeln. Die ausgewählte Person arbeitet an einem konkreten Forschungsprojekt mit und wird im Erfolgsfall Koautor. Die Bewerbungs-Deadline für die ARI Fellowship 2018 endet am 30. September 2016 (für 2017 sind die Fellowships bereits vergeben).

Die Fellowship dauert 12 Monate, in Ausnahmen 6. Das Stipendium beinhaltet die finanzielle Unterstützung der Aufenthaltskosten. In der Bewerbung sollte konkret benannt werden, in welchem der Forschungsfelder des ARIs das Interesse an einer Mitarbeit besteht.

Mehr Informationen und alle erforderlichen Bewerbungsunterlagen sind auf der Homepage des ARI erhältlich https://www.aofoundation.org/ Structure/research/exploratory-appliedresearch/research-institute/medicalresearch-fellowship/Pages/fellowshipapplication

oder über Frau Sonia Wahl, Tel. +41/81/4142541, E-Mail: Sonia.Wahl@ aofoundation.org

\section{Prof. Dr.-Ing. Georg Duda \\ Research Officer}

Julius Wolff Institute and Center for

Musculoskeletal Surgery

Berlin-Brandenburg Center for

Regenerative Therapies

Charité - Universitätsmedizin Berlin

Augustenburger Platz 1

13353 Berlin

georg.duda@charite.de

OP-JOURNAL 2016; 32: 71

(c) Georg Thieme Verlag KG Stuttgart · New York DOI http://dx.doi.org/10.1055/s-0042-108375

\section{Ausschreibung Wissenschaftspreis 2016}

Für den diesjährigen Preis werden noch Bewerbungen bis zum 31. August 2016 entgegengenommen. Der Preis wird am 26. Oktober auf dem AOTrauma Deutschland Symposium im Rahmen des DKOU vergeben, auf dem die Arbeit präsentiert werden darf. Das Preisgeld beträgt 7500 EUR.

Voraussetzungen sind:

- Die Arbeit muss bereits in einem Peerreviewed Journal publiziert sein.

- Das Thema der Arbeit muss im Tätigkeitsbereich der AOTrauma, AOCMF oder AOCMF liegen.

- Es können sowohl klinische Forschungsarbeiten, Grundlagenforschung, systematische Reviews als auch Metaanalysen eingereicht werden.

- AO-Mitgliedschaft (Trauma, CMF oder VET)

- Anwesenheit auf der Sitzung während des DKOU

Einzureichen sind: die Arbeit, ein Motivationsbrief und der Lebenslauf. Das Bewerbungsformular kann von der Homepage der AOTD heruntergeladen werden unter www.aotrauma.org/deutschland

\section{Ausschreibung Nachwuchsförderung}

Die deutsche AO fördert den klinischen Nachwuchs in klinischer und experimenteller Forschung durch Mentoring und eine Anschubfinanzierung. Junge (bis zum Alter von 35 Jahren), klinisch aktive Unfallchirurgen, die in Deutschland an Universitätskliniken, regionalen und kommunalen Krankenhäusern tätig sind, können sowohl klinische als auch experimentelle Forschungskonzepte aus den Bereichen Traumatologie, MKG und VET einreichen.

Bis zum 31. August 2016 können Anträge eingereicht werden. Es werden 6 Arbeiten zur Vorstellung beim DKOU ausgewählt und nach Berlin eingeladen. Die Antragsteller werden eingeladen, ihre Arbeiten auf der Auswahlsitzung am 28. Oktober 2016 vorzustellen und $\mathrm{zu}$ verteidigen. Am Ende der Sitzung werden 3 der eingeladenen Personen mit einer Anschubfinanzierung in Höhe von bis zu 12000 EUR für ihre Arbeit prämiert.

\section{Bewerbungskriterien}

- Kliniker

- Nachwuchs (bis 35 Jahre)

- Unterstützung vom Chef

- AO-Mitglied

- Anwesenheit auf der Auswahlsitzung auf dem DKOU

Alle weiteren Informationen inkl. des Antragsformulars finden Sie auf der Homepage der Deutschen AO: www. aotrauma.org/deutschland 